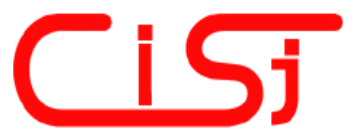

\title{
A GENETIC BASED TECHNIQUE FOR THE DETERMINATION OF POWER SYSTEM TOPOLOGICAL OBSERVABILITY
}

\author{
S. Vázquez-Rodríguez, R. J. Duro \\ Grupo de Sistemas Autónomos, Universidade da Coruña, \\ svr@cdf.udc.es, richard@udc.es
}

\begin{abstract}
In this paper we have addressed the problem of observability of power systems from the point of view of topological observability and using genetic algorithms for its determination. The objective is to find a way to determine if a system is observable by establishing if a spanning tree of the system that verifies certain properties with regards to the use of available measurements can be obtained. To this end we have developed a genotype-phenotype transformation scheme for genetic algorithms that permits using very simple genetic operators over integer based chromosomes which after a building process can become very complex trees. The procedure was successfully applied to standard benchmark systems and we present some results for one of them.
\end{abstract}

Keywords: - Manufacturing plant control, evolution, genetic algorithms

\section{INTRODUCTION}

Electrical power transportation networks are in charge of moving energy from generating points to consumer points, also called loads. For technical and economical reasons, this transportation is carried out by means of high voltage lines which conform a mesh network that can be as large as hundreds or thousands of kilometers, The beginning and end of these lines constitute the nodes of the networks. Thus, we can talk about networks with a number $n$ of nodes and a number $r$ of branches that connect them.

The management of a large electric power system requires detailed knowledge of what is happening in each one of its nodes (power sources, power drains) and branches (power lines connecting nodes) in any given instant of time so that efficient use of the resources can be made and incidences detected. The knowledge about the networks is obtained by means of state estimation techniques based on statistical inference using a set of redundant measurements. The measurements that are usually considered are voltages in the nodes, active and reactive power flow in the branches and power injection in the nodes. There is a non linear relationship between the measurements obtained (z) and the state of the system (x). This relationship is affected by the inherent errors of the measurement equipment (e)

$$
\mathbf{z}=h(\mathbf{x})+\mathbf{e}
$$

Obviously, for the system to have a solution for $\mathbf{x}$, the set of measurements must be large enough and their locations appropriate for the system to be solved (rank of $h$ must be at least as large as the size of $\mathbf{x})$. When these conditions are met the system is said to be observable.

Determining the observability of a system is not a simple task in large power systems. Two main approaches have been followed in the literature in order to address it. On One hand, some authors have resorted to numerical methods trying to determine the range of the Jacobian matrix corresponding to $h(\mathrm{x})$. Within this group we may find [1][2][3]. On the other hand, and due to the precision problems of numerical methods when operating over such large systems, some authors have proposed approaches based on graphs of the power systems to determine its observability [4][5][6][7]. These methods have been called topological methods. The basic idea of topological methods for determining observability is based on the seminal result by Krumpholz and col [4] whereby a system can be said to be topologically observable with respect to a measurement set consisting of one voltage magnitude measurement and paired $\mathrm{P}, \mathrm{Q}$ measurements if and only if there exists a spanning tree of the system of full rank. In practical terms, we need to find a spanning tree that contains all the nodes in the network and where the branches have measurements assigned to them. To this end, the measurements that are available in the network are assigned to branches following two criteria: The power measurements in the branches of 
the graph are assigned to the branches of the tree corresponding to their location. The node power measurements are assigned to one (and only one) of the branches that converge in the node. It is important to take into account that a measurement can only be used once in the construction of the tree.

To address the problem of finding this spanning tree many heuristic solutions have been proposed such as [5][6][7], they usually differ in the algorithm they use for assigning node measurements to branches when constructing the tree, but their basic procedure is very similar in terms of reading the network sequentially and constructing a tree in a stepwise fashion. As with most tree construction techniques, these approaches are very prone to getting stuck, that is, running out of possible paths before achieving a full rank tree due to previous inadequate selections of branches to follow in the power system graph. As a consequence, backtracking is usually required, which in the extreme case leads to a random search. This problem becomes even more poignant due to the quasiexponential growth of possible trees as the size and/or connections of the power system grow.

Due to the problems of classical heuristic methods, we have decided to seek other ways of implementing the exploration procedure in the possible tree space. In fact, the problem of determining the spanning tree of full rank can be transformed into an optimization problem where the fitness criterium is related to some desired features of the final tree, such as maximum number of nodes or maximum use of branch measurements. Different optimization techniques may be employed in order to determine the tree with maximum fitness, but due to the imprecise nature of the fitness function and to the complexity of the search space for large power systems, in this paper we have chosen a different approach by using evolutionary techniques for the determination of the spanning tree of full rank.

\section{EVOLUTION AND ENCODING}

Some authors have already made some inroads in the use of evolutionary technique in the field of topological observability [8][9]. From their work it can be deduced that there are three hurdles to overcome in the use of a genetic algorithm based solution to determine topological observability:

- The encoding of the trees.

- Defining the appropriate genetic operators and fitness function.

- Reigning in sufficient computing power to be able to solve large problems.
In this paper we will concentrate on the first two and the third one will be considered elsewhere.

Encoding valid trees that correspond to the power system we are considering is a difficult problem as there are many restrictions to the way in which they can be constructed. Consequently, if the encoding is not carefully handled, most of the chromosomes would represent invalid solutions, that is, trees that do not represent the network and/or trees that do not correspond to an observable solution, especially after mutations or crossovers. To verify all the constraints and generate trees that are always valid, in this work we have chosen an indirect representation of the phenotype in the chromosome. Our chromosomes do not represent the tree, but rather the way in which we construct the tree from the information on the real power system. Thus, when constructing a tree from the system we first obtain a connectivity matrix where we represent the connections between the nodes of the power system and whether these connections or the corresponding nodes have measurements. Once this matrix is constructed we can define the maximum connectivity $\mathrm{C}_{\max }$ as the number of connections of the node with maximum number of real connections. The chromosome that encodes the possible trees representing the power system will have a length of $2 n$ integers, the first one between 1 and $n$ and the rest with values between 0 and $C_{\max }$ where $n$ is the number of nodes in the power system.

The chromosome may be written as $\mathrm{P}_{1}, \mathrm{~F}_{1}, \mathrm{P}_{2}, \mathrm{~F}_{2}, \ldots . \mathrm{P}_{\mathrm{n}}, \mathrm{F}_{\mathrm{n}}$. where $\mathrm{P}_{\mathrm{i}}$ represents the relative number of the node and $F_{i}$ the fan out of this node (number of branches departing from it). Thus, if we want to construct a tree from the chromosome we take $\mathrm{P}_{1}$ and go to the corresponding line in the connection matrix. We take a $F_{1}$ (fan out value) and it will tell us the fan out of this node (number of branches originating in it). If this number is larger than $C_{i}$ (number of possible connections for this node at this time), we take as effective fan out $F_{i}{ }_{i}^{\text {eff }}$ $=\mathrm{F}_{\mathrm{i}} \bmod C_{\mathrm{i}}$ otherwise $\mathrm{F}_{\mathrm{i}}{ }^{\text {eff }}=\mathrm{F}_{\mathrm{i}}$. The following $\mathrm{F}_{\mathrm{i}}$ eff pairs in the chromosome will correspond to the nodes connected to node $i$. Whenever we use a node or a measurement it is eliminated from the connection table. This process is carried out recursively until the whole tree is constructed or a situation where no more nodes are available in the connection matrix lines corresponding to the leave nodes of the tree.

Some comments must be made on what type of measurements we are considering and how they must be chosen in order to verify all the requirements of observability. As indicated before there are basically two types of measurements, node measurements and branch measurements. A branch measurement can only be used in a branch of the 
tree that connects the same two nodes that are connected in the original power system by this branch. Consequently, branch measurements are only usable in their branch and must thus be used as the measurement for that branch if it appears in the tree, even if there are node measurements available for the same branch. On the other hand, a node measurement can be used for any branch that links the node with the measurement to any other node, however, for the tree to be a valid representation for observability, it can only be used in one of these branches. Consequently, when a node measurement is used in the tree it becomes unavailable for further use. Summarizing, when two nodes are connected by means of a branch of the tree, if

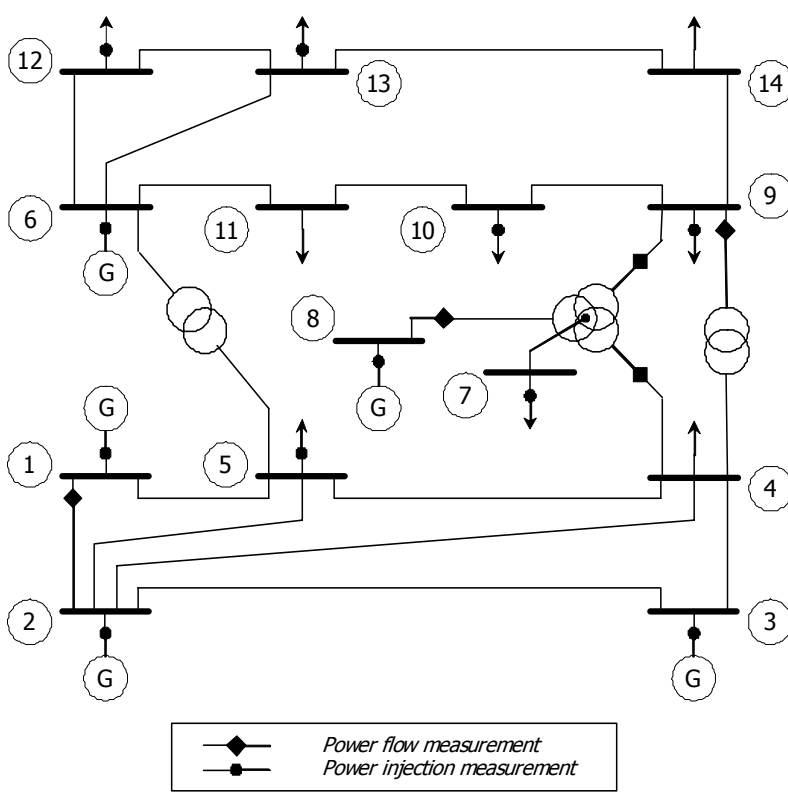

Fig. 1 - Basic unifilar representation of the IEEE 14 node benchmark network.

there is a branch measurement between these two nodes, this is the measurement assigned to the branch. If there is no branch measurement available, but the parent node has a node measurement, this is the one that will be used. Finally, if only the child node in the tree has a node measurement, it will be the one chosen.

This strategy for encoding trees in chromosomes provides a way to use a very simple genotype $(2 n$ integer values for a power system with $n$ nodes) that always results in valid trees. Thus, now all the genetic algorithm has to do is to find one of these chromosomes that produces a whole tree, that is, a tree that contains all of the nodes in the power system. To do this we will define a very simple fitness function that increases with the number of nodes in the resulting tree with a modulating term that increases with the percentage of nodes outside the tree that contain node measurements.
As a result of the simplicity of the genotypic encoding, the genetic operators that are used are quite straightforward. Take into account that we are handling integer value strings. Thus, crossover is a classical two point crossover and mutation is a simple random mutation. The only element we must

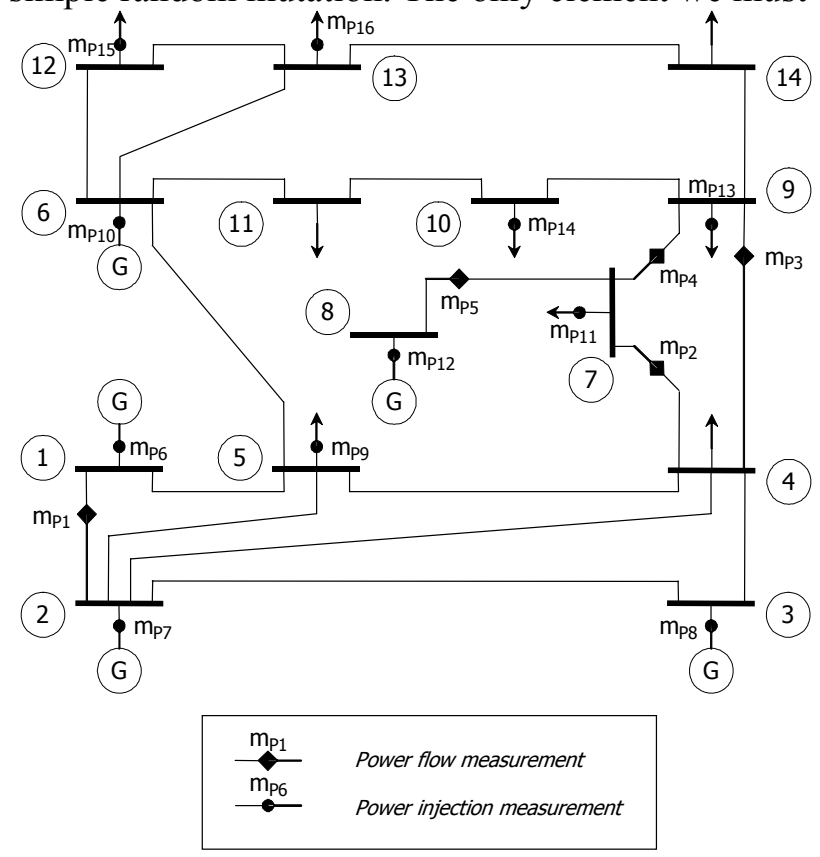

Fig. 2 - Transformation of the basic representation of the IEEE 14 node benchmark network into a node set where $\mathbf{G}$ indicates generator, an arrow a consumer, and the node (power injection) and branch (power flow) measurements are indicated.

take into account is the significance of mutations, a mutation in a gene in the left part of the chromosome produces more drastic changes in the resulting phenotype than one in the right part of the chromosome and this is taken into account through a positional weight term for the mutation.

\section{EXPERIMENT}

The procedure presented above was used on one of the classical benchmark problems in power system observability, the IEEE 14 node system. This system, in terms of its electrical unifilar diagram, representing the real topology of the network and certain parameters characterizing the equipment and transport lines, is shown in Fig.1. In this diagram, the nodes are represented by thicker lines and are numbered from 1 to 14 . Different elements are connected to these nodes, such as lines, loads (represented by arrows) and generators (indicated by a G).

Given the fact that both generation and consumption occurs at lower voltage levels than those used for transportation it is necessary to include power transformers to adapt the voltage levels, and which are included between the lines in the figure. 
As this representation is too cumbersome for our purposes, we have directly obtained a simplified

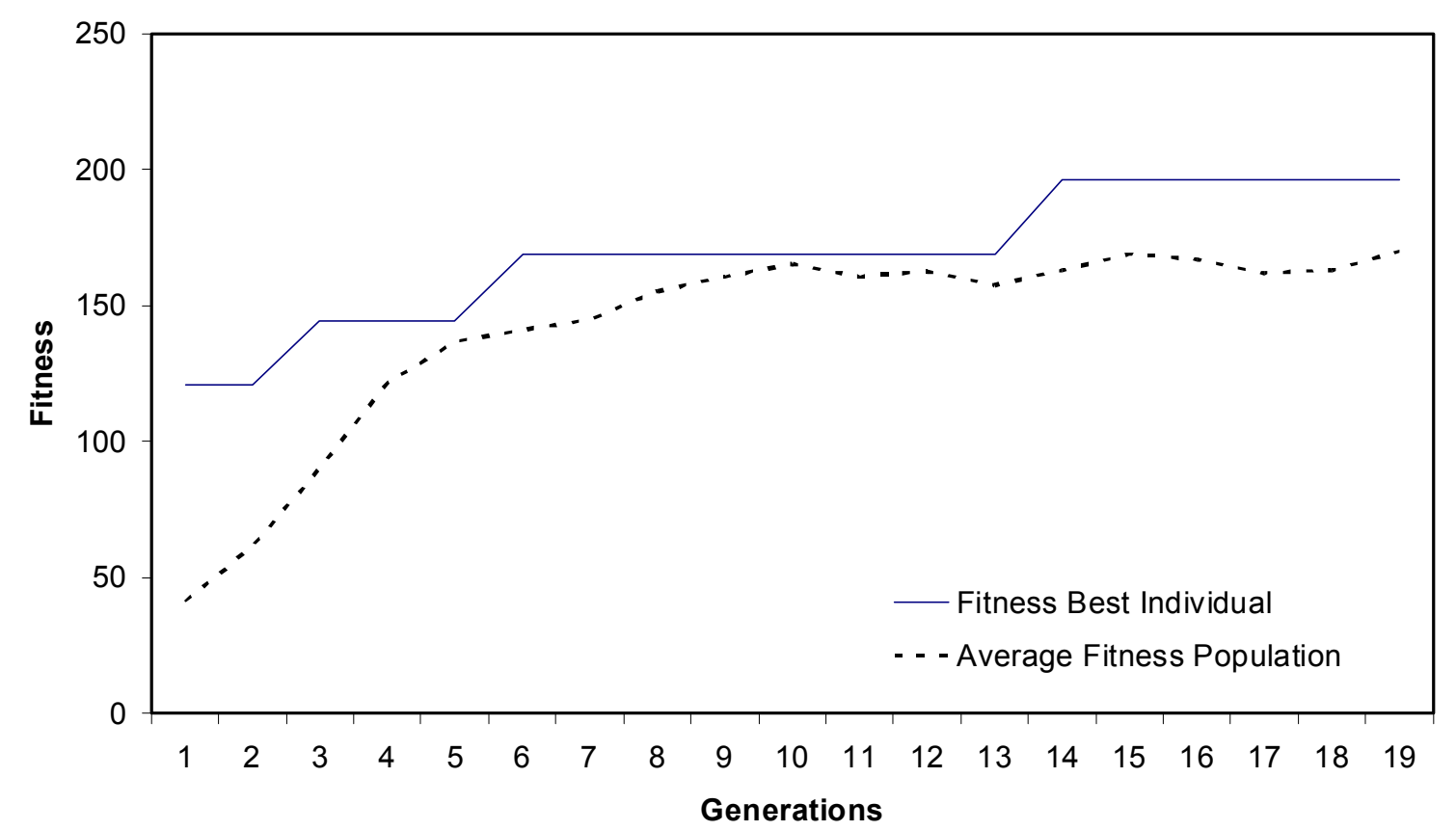

Fig. 3 - Fitness of the best individual (solid line) and average fitness of the population (dashed line) in the evolution of the IEEE 14 node problem. Fitness of 196 corresponds to full spanning tree.

(Fig.2), and which is the one we are going to work with. As observability has to do with the measurements obtained from the system their nature and their positions, the available measurements have been indicated in the figure. These have been divided into two groups, those that evaluate the power that flows in the lines and those that measure the power generated or consumed in a node. The genetic algorithm employed is a classical with a two point crossover operator and a random mutation operator where smaller changes in the genes were favoured in order to improve exploration around previous points. Tournament selection was used with a 2 individual tournament window so as to reduce pressure and prevent premature convergence as much as possible.

The idea here was to obtain good results fast but with the minimum possible computations and avoiding premature convergence. The use of very small populations would induce premature convergence of the population and large populations would unnecessarily increase the computational load. We finally settled for a population of 40 individuals, a crossover probability of 0.9 , a mutation probability of 0.2 and an elitist strategy where the best individual for one generation was preserved. The fitness function employed was a very simple one, just the square of the number of nodes of the tree with the maximum number of nodes out of the trees generated for a given chromosome. Take into account that the objective is to obtain a single tree that contains all the nodes, but while evolution has not achieved the optimal, each chromosome leads to a forest of trees, each one of them containing a smaller number of nodes.

\section{RESULTS}

The evolution of the fitness for the best individual each generation as well as the average fitness for the whole population are presented in Fig.3. It must be taken into account that a complete spanning tree for this system would imply a fitness value of 196. The figure clearly shows that the choice of parameters is adequate. As evolution progresses the fitness of the best individual increases and, even though the average fitness of the population comes close to that of the best individual in some points it never quite prematurely converges to it, preserving enough variety to allow for continued evolution up to the optimal.

This figure also shows that the optimal is achieved, that is, a spanning tree containing all the nodes is obtained in about 14 generations, or something like a few seconds on a desktop PC. In fact, more than one solution for the problem is obtained in the population. Figures 4 and 5 show two of the solutions obtained by the system. In these figures we indicate using thick lines the connections used by the algorithm in order to construct the tree as well as the measurements employed in each case. It is very important to point out that all of the solutions obtained were valid trees compatible with the network and that they all verified all the constraints imposed. This validates the use of a 
complex encoding scheme that leads to a very operate with within the evolutionary algorithm. simple genotypic expression that is very easy to

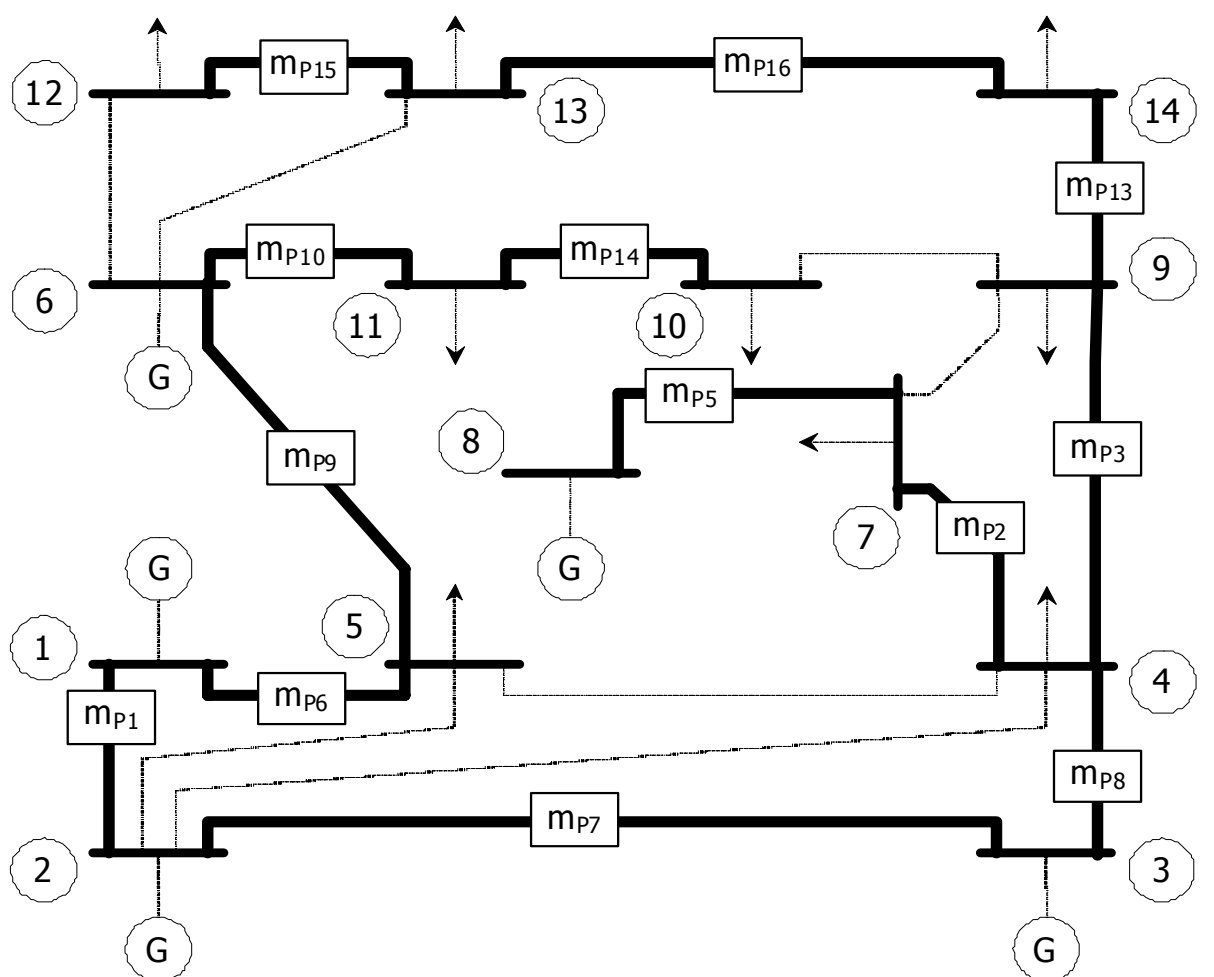

Fig. 4 - One of the complete spanning trees obtained using the strategy proposed for the IEEE 14 benchmark.

\section{CONCLUSIONS}

In this paper we have presented a way to decide if a power network is observable through the use of the concept of topological observability and employing genetic algorithms. The most important characteristic of the procedure has to do with the way the chromosome is encoded. The encoding procedure, which basically encodes the procedure for constructing the tree from the network, provides the means to work with very simple integer valued genotypic expressions that are quite easy to manipulate in a genetic algorithm, and at the same time does not present any limitation on the trees that can be constructed and guarantees that all the trees and forests produced will be topologically and electrically valid.

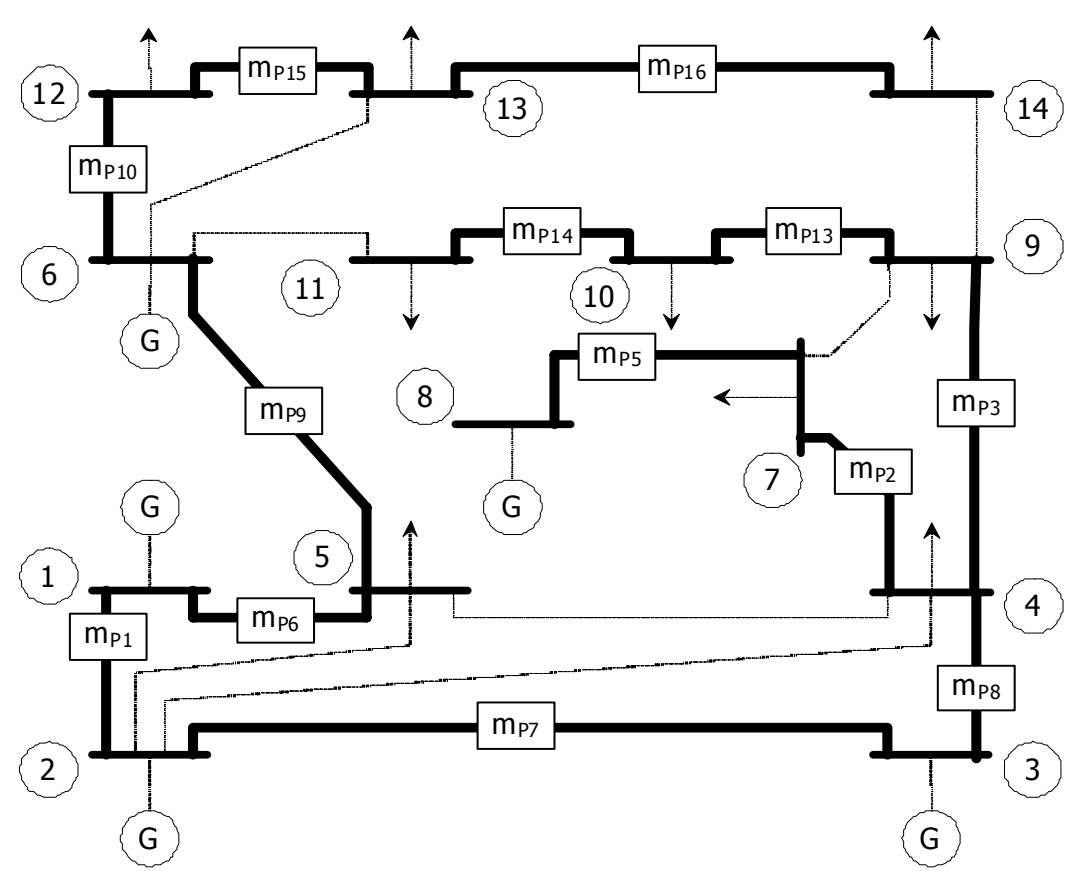

Fig. 5 - Another complete spanning trees obtained using the strategy proposed for the IEEE 14 benchmark. 


\section{ACKNOWLEDGEMENTS}

This work was partially funded by This work was funded by Xunta de Galicia under project PGIDIT02PXIB10501PR the MCYT of Spain under projects TIC2000-0739C0404 and NATO under PST.CLG.978744.

\section{REFERENCES}

[1]A. Monticelli, F. F. Wu. Observability analysis for orthogonal transformation based state estimation, IEEE Transactions on Power Systems, Vol. PWRS-1, No. 1, Febr. 1986. pp. 201-208.

[2]F. F. Wu, W-H. E. Liu, S-M Lun. Observability analysis and bad data processing for state estimation with equality constraints, IEEE Transactions on Power Systems, Vol. 3, No. 2, May 1988. pp. 541-548.

[3]F. F. Wu, W-H. E. Liu, L. Holten, A. Gjelsvik, S. Aam. Observability analysis and bad data processing for state estimation using Hachtel's augmented matrix method, IEEE Transactions on Power Systems, Vol. 3, No. 2, May 1988. pp. 604-611.

[4]G. R. Krumpholz, K. A. Clements, P. W. Davis. Power system observability: a practical algorithm using network topology, IEEE Trans. Power Apparatus and Systems, Vol. PAS-99, No. 4, July/Aug. 1980. pp. 1534-1542.

[5]K. A. Clements, G. R. Krumpholz, P. W. Davis. Power system state estimation with measurement deficiency: an algorithm that determines the maximal observable subnetwork, IEEE Trans. Power Apparatus and Systems, Vol. PAS-101, No. 9, Sept. 1982. pp. 3044-3052.

[6]V. H. Quintana, A. Simoes-Costa, A. Mandel. Power system topological observability using a direct graph-theoretic approach, IEEE Trans. Power Apparatus and Systems, Vol. PAS-101, No. 3, March 1982. pp. 617-626.

[7]Hiroyuki Mori, Senji Tsuzuki. A fast method for topological observability analysis using a minimum spanning tree technique, IEEE transactions on power systems, Vol.6, No.2, May 1991. pp. 491-500.
[8]H.Mori, H.Tanaka. A genetic approach to power system topological observality. Proc. IEEE International Symp. on Circuits and Systems, N.Y. 1994, pp.1141-1144.

[9]H. Mori. Power System Topological Observability Index and Maximization with a Genetic Algorithm. Proc. of 1993 International Symposium on Nonlinear Theory and Its Application (NOLTA'93), Dec. 1993, pp. 10691072.

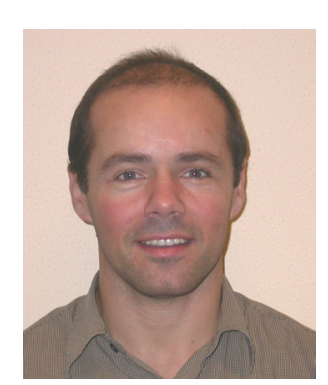

Santiago VázquezRodríguez received a M.S. in Industrial Engineering from the University of Vigo, Spain in 1990. He has worked in different private industries designing different installations for the automotive, energy and gas industries. He is currently Profesor Titular de Escuela Universitaria in Electrical Engineering at the University of A Coruña. His main research interests are related to the analysis of power systems and evolutionary algorithms.

Richard J. Duro received the B.S. and M.S. degree in Physics from the University of Santiago de Compostela, Spain, in 1989, and a PhD in Physics from the same University in 1992. $\mathrm{He}$ is currently a Profesor Titular in the Department of Computer Science and member of the

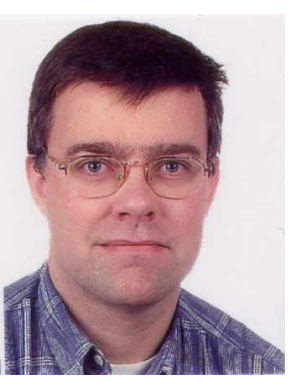
Autonomous Systems Group at the University of $A$ Coruña, Spain.His research interests include higher order neural network structures, signal processing and autonomous and evolutionary systems. He is a member of the IEEE. 